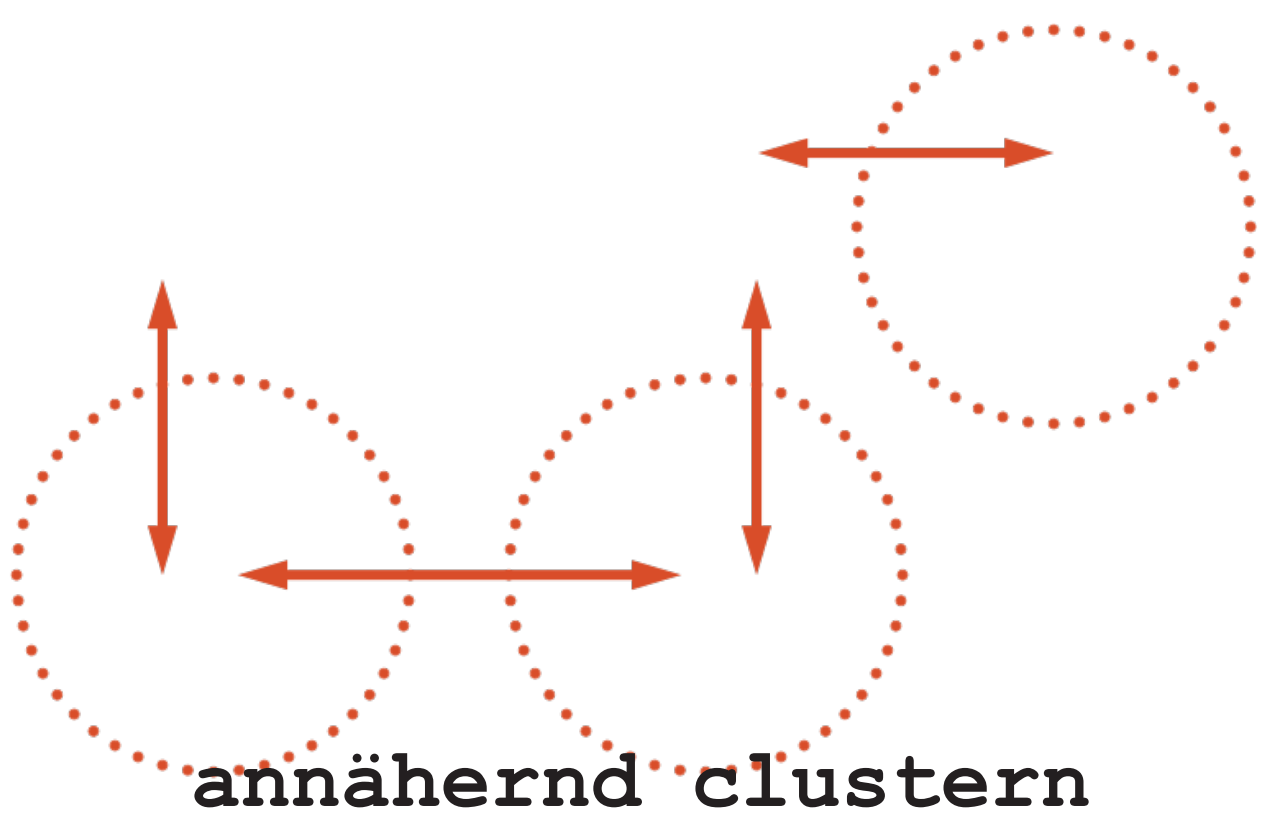

sich bestmöglich aufeinander beziehen

\title{
Michael Zinner
}

mit Dank für Resonanz an Ulrike Schmidt-Zachl und Katharina Lenggenhager herausgegeben von Michael Zinner, schuIRAUMkultur an der Kunstuniversität Linz redigiert von Siegfried Kraus

für das Titelbild @2020 schulRAUMkultur mit Dank an Andrea Hilmbauer online verfügbar unter https://doi.org/10.35468/nAB2020-413 (CC-Lizenz BY-NC-ND) gefördert vom EU-Programm Erasmus+, Projekt PULS+2017-1-AT01-KA203-035056 mit Stand von 22.12.2020 
Diese Notiz vertieft das Verständnis von sogenannten „annähernden“ Clustern, ohne atmosphärisch-materielle Dimensionen von Architektur zu berücksichtigen. Michael Zinner zieht dazu vier zeitgenössische Schulbauten, die in den letzten zehn Jahren umgebaut und/oder saniert wurden, als Beispiele heran. Entlang metaphorisch beschriebener Kraftflüsse im Kreis, in Bezug auf qualitative und quantitative Aspekte der Mitte und gemäß dreier Dimensionen einer Untersuchung - geometrische Gefügequalitäten, physiologische Hautqualitäten und unterschiedliche Nutzungsoptionen - spürt er den Gemeinsamkeiten und Unterschieden dieser Schulen nach. Er plädiert für ein genau differenziertes Analysieren der einzelnen Fälle. Dementsprechend zeigt Zinner abschlieBend, wie nuancierte Details ein Gelingen annähernder Cluster auf vielfältig unterschiedliche Weise und jenseits erster Blicke mitbegründen.

\section{das Thema}

In einem „annähernden Cluster" sind Klassenräume bestmöglichst um eine - meist nicht explizite - Mitte (eine "annähernde Mitte") herum gruppiert. Die Idee des Clusters kann durch Maßnahmen in der GefügeGeometrie, in der Wand-Durchlässigkeit oder in der Möbel-Dienlichkeit angestrebt bzw. lediglich „annähernd“ erreicht werden. Lernräume können dann so gut als möglich zueinander in Beziehung stehen, um beispielsweise ein Arbeiten in unkompliziert wechselnden GruppengröBen, eben auch klassenübergreifend, zu erlauben.

Die Idee, sich gewissermaßen im Kreis inklusiv auf Augenhöhe zu begegnen, wird in bestehenden Bauwerken (meist) nur bedingt möglich. Insofern greift hier die metaphorische Annäherung (vgl. Zinner $\rightarrow$ nAB410; $\rightarrow$ nAB411; $\rightarrow$ nAB412) nur mehr in Teilen bzw. rudimentär, auch wenn immer wieder von "Mitten" und „Rändern" als potenzielle bzw. eben „annähernde" Qualitäten im Gefüge gesprochen werden kann.

Die Montag Stiftung Jugend und Gesellschaft $(2017,27)$ haben in ihrem Grundlagenbuch „Leitlinien für leistungsfähige Schulbauten in Deutschland“ mit "Klassenraum plus" einen Begriff geprägt, der gewissermaßen zwischen Gangschule und Cluster steht. Das „plus"-Prinzip wird hier als Erweiterung in die Erschließung oder in zusätzlich, meist kleinere Räume daneben verstanden (ebd.). Bis zur Richtgröße von rund $75 \mathrm{~m}^{2}$ (ebd.) werden wenige bis keine Möglichkeiten gesehen, in einem Klassenraum vielgestaltiges Lernen gelingen zu lassen.

Für ungestörten Unterricht in der Klasse sind vielmehr Gangschulen prädestiniert und leistungsstark. Für den steten und einfachen Wechsel von Lernanordnungen (Einzelarbeit, Arbeiten in Kleingruppen, Instruktion für Klassen, Diskussion im Sitzkreis) hingegen sind Gangschulen mit ausschließlichen Anordnungen für die Instruktion mit hoher 
Wahrscheinlichkeit hinderlich - meist zu wenig aufeinander bezogen die Klassenräume, meist zu wenig beweglich die Möbel, meist zu wenig durchlässig die Wände. Bei Sanierungen bzw. Umbauprojekten wird daher immer öfter entweder von der Schule, von der Schulverwaltung oder vom Architekturbüro (oder von einer Kombination dieser drei) die Figur eines Clusters als Prinzip angestrebt. Doch nicht selten müssen bei Gangschulen in Bezug auf die Geometrie des Gefüges und auf die Beschaffenheit der Wände (weniger auf die Optionsvielfalt der Einrichtung) Kompromisse eingegangen werden, sodass ein Cluster eben lediglich "annähernd" gelingt.

Bauwerke können "schneller" sein als Schulen - und umgekehrt. Einmal, wenn ein Architekturbüro mit einem sich der Figur des Clusters bedienenden Schulumbau eine "schlafende Schule" (heraus)fordert. Und ein zweites Mal, wenn eine Schule mit weit gereiften pädagogischen Ambitionen im Cluster in einem "schlafenden Gebäude" in Form einer überalterten Gangschule haust. In beiden Fällen kann das zu improvisierten Nutzungen führen. Im ersten Fall kann die physische Welt die soziale, im zweiten Fall die soziale Welt die physische überfordern. Einmal werden Öffnungen zwischen Räumen beispielsweise zugeklebt und dann wieder müssen sperrige Möbel am Gang für eine personalisierte Lernpraxis arrangiert werden. Die kreativ-erfinderische Kraft "wacherSchulen", die ihre ungewohnte Schulpraxis trotz gewöhnlichem Bauwerk umzusetzen versuchen, ist beispielsweise aus vielen Schulpreis-Portraits bekannt (Robert-Bosch-Stiftung $\mathrm{GmbH}$ o. J.; www.deutscher-schulpreis.de). Und es finden sich mittlerweile auch Fälle "schlafender Schulen", die einen Kraftaufwand betreiben, um ihre gewohnte Schulpraxis trotz eines "ungewohnten" - als ungewöhnlich wahrgenommenen - „wachen Bauwerks" weiterführen zu können.

Gangschulen, die mit Abstand größte Zahl von Bestandsschulen, oszillieren heutzutage gewissermaßen in dieser Spannung von Figur und Praxis. Viele aktuelle Reibungen zwischen Bau und Schule sind insofern eine historisch logische Erscheinung. Sie verdeutlichen auch, warum dialogische Prozesse bei Umbauten oder Sanierungen derzeit Sinn bilden. Und natürlich ziehen nicht immer alle Interessen am gleichen Strang. Aktuell wird daher bei jedem Umbau einer Schule zu entscheiden sein, wie ein baulicher Umbruch zur Schule passen kann oder wie ein pädagogischer Aufbruch ein Bauwerk auf sich anpassen kann. Daher ist es wesentlich zu verstehen, wie bauliche Potenziale bestehender Schulen für aktuelle Erfordernisse pädagogischer Praxen gehoben werden können. 


\section{vier Beispiele}

Das Thema wird in vier Beispielen anschaulich gemacht, die ausgesucht wurden, weil sie verschiedene Ausgangssituationen mit sich brachten. Der Auflistung sind - wie schon in den Notizen nAB 411 und nAB 412 Schulart, Standort und Errichtungszeit des Bauwerks zu entnehmen, auch, wer es entworfen hat. Ich werde ohne viele Fotos analysieren, weil strukturelle Eigenschaften diskutiert werden wollen - auch dieser Text dient dem entsprechenden Architektur-Diskurs jenseits konstruktiver, materieller und atmosphärischer Aspekte, auch wenn sie nicht gänzlich außer Acht gelassen werden können. Umfassendere Besprechungen, die auf architektonische und pädagogische Details und deren Verschränkung eingehen, sind als Notizen der Reihe 800 in Planung.

$\begin{array}{rll}\text { Volksschule Andelsbuch (1960er) } & 2022 & \text { stöckler gruber } \\ \text { Volksschule Deutschfreistritz (1930er) } & 2020 & \text { balloon architekten } \\ \text { Volksschule Angedair (1900er) } & 2018 & \text { Franz\&Sue } \\ \text { Volksschule Donawitz (1920er) } & 2016 & \text { schulRAUMkultur \& nonconform }\end{array}$
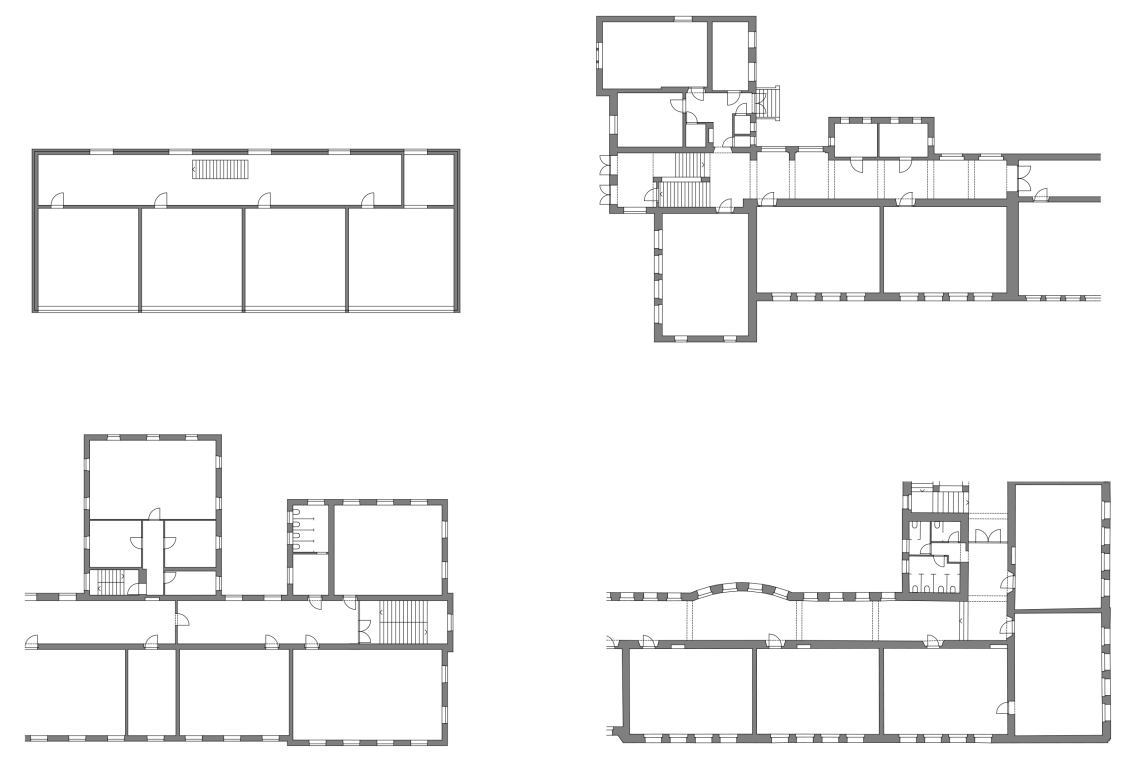

ABB 01

Andelsbuch, Deutschfeistritz, Angedair, Donawitz @2020 schulRAUMkultur

Die Reihung in den Abbildungen erfolgt von links oben nach rechts unten von Andelsbuch, Deutschfeistritz, Landeck-Angedair und LeobenDonawitz. Die Bestandsbauten (ABB 01) sind jeweils dezidierte Gangschulen und sollen durch Umbau-, Zubau- und Sanierungsmaßnahmen zukünftig die Räumlichkeiten der Schule durch Neuordnungen im Be- 
standes durchlässiger gemacht werden. Die beiden Schulen in Deutschfeistritz und Leoben-Donawitz sind Teil größerer Bildungszentren mit je einer Volksschule, einer Mittelschule und einer Polytechnischen Schule, die Schulen in Andelsbuch und Landeck-Angedair stehen für sich. Alle vier Schulen wirken als Massivbauten mit Lochfassaden (Fenster in Wänden), in Andelsbuch sind tragende Wände in Stahlbeton ausgeführt (Nachkriegszeit), in allen anderen Schulen aus Vollziegel gemauert (Gründerzeit).
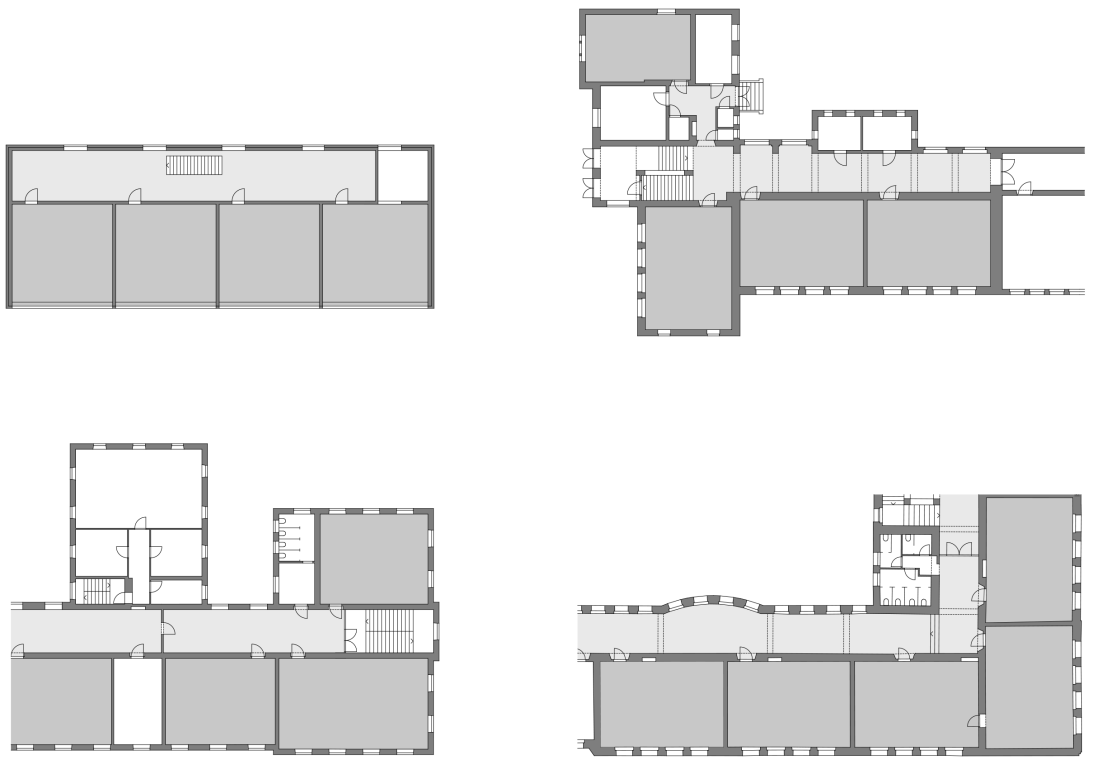

ABB 02 Gänge (hell) und je vier bis fünf Klassen (dunkel) @2020 schulRAUMkultur

In den vier Bestandsbauten zeigen sich ähnliche Gangformationen mit unterschiedlichen Zuordnungen von Räumen (ABB 02). In Andelsbuch erschließt ein geradliniger Gang alle Klassenräume auf einer Seite (Einhüftigkeit), in Deutschfeistritz liegt ein Raum den anderen dreien am Gang gegenüber, in Leoben-Donawitz führt der abermals einhüftige bzw. einbündige Gang um eine 90-Grad-Ecke und in Landeck-Angedair erschließt der Gang in der Mitte des Bauwerks beide Seiten (die Struktur ist zweihüftig bzw. zweibündig). Die größte Länge weist der Gang in Leoben-Donawitz auf (wobei hier nur jene Teile des Gefüges betrachtet werden, die zu einem Cluster umgebaut werden). In Andelsbuch liegt eine einläufige Treppe frei in der Gangfläche, in Deutschfeistriz und Landeck-Angedair erschließt je eine zweiläufige Treppe das Gebäude am Kopfende des Ganges. In Leoben-Donwitz führen beide Richtungen am Gang zu Treppen. In allen Schulen vermittelt exakt je eine Türe vom einzelnen Klassenraum in den Gang. In Landeck-Angedair sind Klassenräume in Gebäudeecken von zwei Seiten belichtet. In Andelsbuch sind sie das über Oberlichten. Ansonsten sind - bis auf vereinzelte Fenster 
in Deutschfeistritz - alle Räume jeweils lediglich von einer Seite belichtet. Entsprechend ihrer Entstehungszeiten sind die bestehenden Klassenräume mit $65 \mathrm{~m}^{2}$ bis $71 \mathrm{~m}^{2}$ in Relation zu aktuellen Maßstäben sehr geräumig.

\section{Andelsbuch 2022}
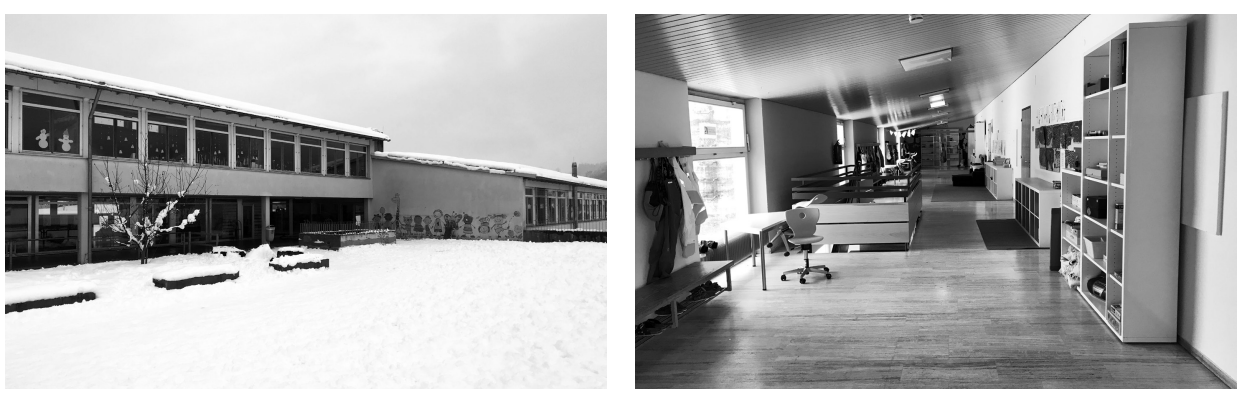

ABB 03

zweigeschoßiges, versetztes Bauwerk, überbreite Gänge @2015 VS Andelsbuch

Die Volksschule in Andelsbuch in Vorarlberg wird ab 2020 saniert, umgebaut und erweitert und soll 2022 fertiggestellt sein (es gibt also noch kein Bildmaterial). Das Projekt von stöckler gruber architekten (www.sgarchitekten.at) nutzt das vorhandene Potenzial der Vierergruppe, denn mit seiner Breite und angemessen geringen Raumhöhe weist der Gang Aufenthaltsqualität auf. Und die in sich ruhenden Klassenzimmer mit acht mal acht Metern und zweiseitiger Belichtung evozieren die Möglichkeit einer von der frontalen Anordnung abweichenden Möblierung. Darüber hinaus unterstützt die Geometrie des Ensembles ein beinahe gleichrangiges gemeinsames Benutzen der Gangfläche (vgl. Zinner $\rightarrow \mathrm{nAB} 412$ ).

Aus Klassenzimmern werden nach dem Umbau "Lernateliers" und aus dem Gang wird eine "Lernlandschaft". Die weiterhin bestehende Treppe und der neu hinzukommende Aufzug irritieren mit ihren Lagen allerdings die Wohnlichkeit der dann neuen Mitte, sodass nicht von einem "seitigen Cluster", sondern von einem „annähernden Cluster" gesprochen werden sollte. Der „annähernden Mitte" fehlt die erforderliche Tiefe und dem gesamten System entsprechende Zusatzräume wie Räume für Gruppen und Team (Toiletten und Garderoben sind im Geschoß darunter untergebracht). 

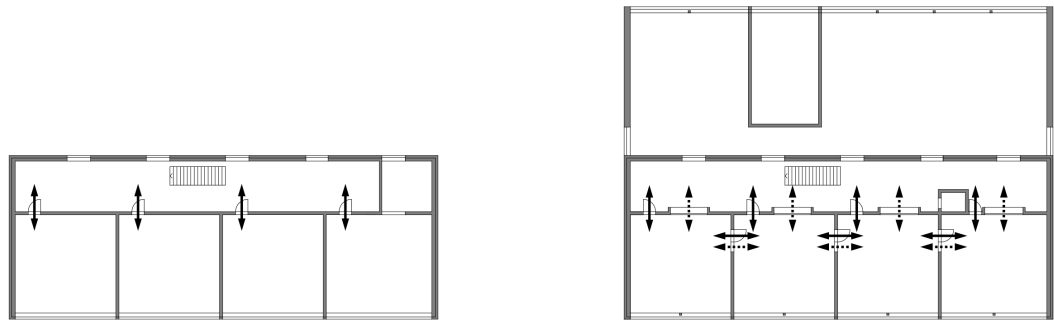

ABB 04 von vier zu sieben Türen plus vier Erkerfenster @2020 schulRAUMkultur

Im Vergleich zwischen Bestandsbau und Umbauprojekt (bAIK 2019), mit einem in der Abbildung dargestellten Luftraum zentraler Flächen des Erdgeschoßes, liegen Maßnahmen zur Erhöhung der „Beziehungsfähigkeit" der Räume vor allem in einer fast Verdreifachung der Anzahl der Wandöffnungen. Je Lernatelier wird eine Tür zum Nachbarzimmer in die tragende Stahlbetonwand geschnitten. Und mit einem zusätzlichen erkerartigen Fenster öffnet sich jedes Lernatelier im Ausmaß von einem Drittel der Wand zur Lernlandschaft hin. Im Zusammenwirken von Blickbeziehungen und Lichtführungen entsteht ein stetiger Raumfluss (Oberlicht über der Mittelwand für die Lernateliers und Durchblick quer durch den gesamten Gebäudeteil).

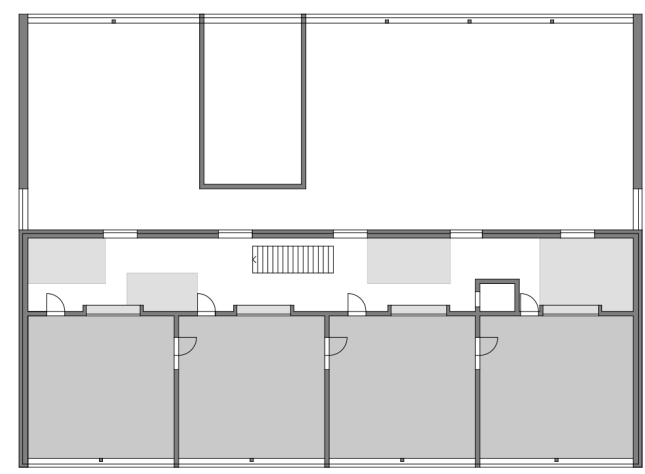

ABB 05 vier Lernräume und ein „Lerngang“ mit Durchwegung @2020 schulRAUMkultur

Neben dieser baulichen Steigerung der „Atmungsaktivität” des Systems gilt es, zukünftig leicht veränderbare Möblierung auszuwählen. In der Gefüge-Ordnung sind neuralgische Punkte wie bestehender Treppenlauf und neuerrichteter Aufzug von besonderer Bedeutung. Denn die verstärken die Erschließungsfunktion der Mitte, die vorrangig Lernlandschaft sein soll. Und sie gewichten die vier Lernräume über die übliche Reihung Anfang-Mitte-Ende hinaus. So entsteht hinter dem Aufzugsschacht eine intime Situation für eine Lerngruppe, die Exklusivität ausstrahlt. Und um die Treppe entsteht in der Mitte der Mitte ein für die Nutzung eingeschränkt verfügbarer Bereich, der ein hohe öffentliche Bezüglichkeit aufweist. Doch diese spezifischen Stellen im System 
können Sinn erfahren, wenn die pädagogische Konstellation angepasst bzw. dafür entwickelt wird - oder wenn sie sich der baulichen Struktur „annähert".

\section{Deutschfeistritz 2020}
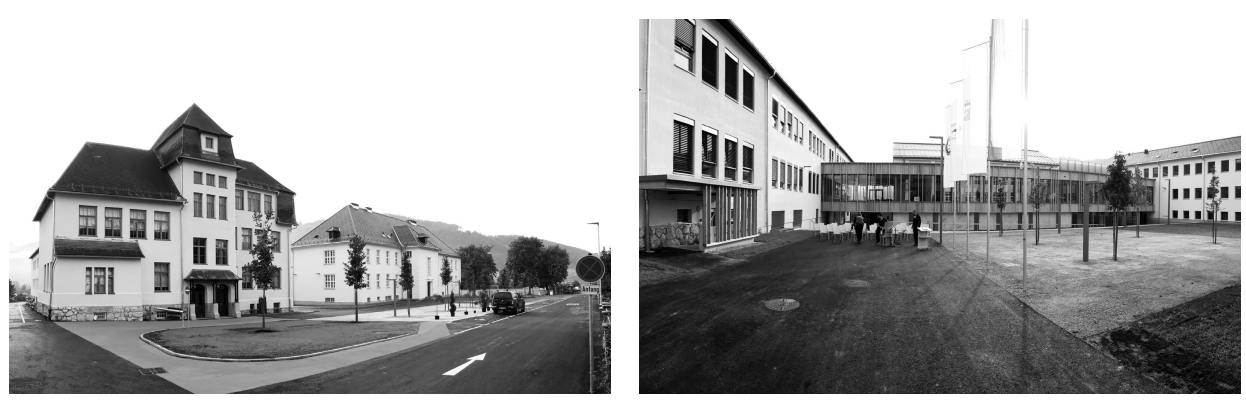

ABB 06

Bauwerk aus den 1930er Jahren und gemeinsamer Schulhof @2020 Michael Zinner

Die Volksschule in Deutschfeistritz ist im Rahmen der zweiten Bauetappe für das Schulzentrum des Ortes bis 2020 umgebaut, erweitert und saniert worden. Der Bauteil, der hier betrachtet wird, steht für sich, wirkt romantisierend und ist in ein Schulgeviert, das hauptsächlich aus Bauten der Nachkriegszeit besteht, rund um einen großzügigen Schulhof eingebettet (ABB 06).

Das bestehende Gebäude ist mit dem mittig liegenden und über drei Meter breiten Gang auch ein Punktbau, nicht nur eine lineare Struktur. Er wird erst im Kontext mit einem Zubau aus der Nachkriegszeit zu einem uninspiriert langen Schulgebäude mit endlos wirkenden Gangfluchten. Im Inneren der Volksschule „bremsen“ Gewölbeformationen, Gurtbögen, seitliche Pfeiler und Vor- wie Rücksprünge die Gangflucht. Zwei der mit $71 \mathrm{~m}^{2}$ sehr geräumigen Klassenräume verhalten sich in Bezug zum Gang gleichrangig konform, der dritte liegt im Kopfbau und ist um $90 \mathrm{Grad}$ verdreht. Ihm gegenüber befindet sich eine ehemalige Wohnung (mit eigenem Eingang). Wesentliche Maßnahmen zur Erhöhung der "Beziehungsfähigkeit" betreffen abermals die Steigerung der Anzahl an Wandöffnungen von ehemals vier Türen auf sieben und zusätzlich das Ein(ge)fügen dreier Fenster (ABB 07). 

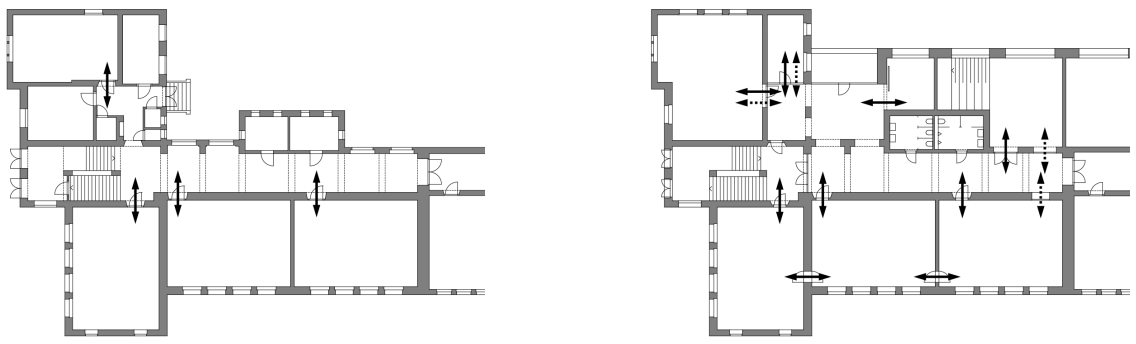

ABB 07 von vier zu sieben Türen plus drei Fenster @2020 schulRAUMkultur

Für den Wunsch nach einer Clusterschule erweitert das Büro balloon architeken (www.balloon-rgw.at; bAlK 2016) die Grundrissflächen (ABB 08), nimmt einen weniger belichteten Mittelgang in Kauf und gewinnt einen Marktplatz (links in der Mitte) mit Balkon, einen Gruppenraum und eine Lern-Bibliothek (rechts oben) dazu, die darüber hinaus mit dem zweiten Cluster im Obergeschoß durch eine Lese-Arena vertikal verbunden ist. Wenn diese Bibliothek auch als Teamraum eingesetzt wird (was denkbar ist), sind alle Räume, die ein Cluster aufweisen kann, vorhanden (Garderoben befinden sich in unmittelbarer Nähe). Das Bauwerk insgesamt konzentriert sich dann noch stärker auf sich selbst und forciert das bereits bestehende Wesen des Punktförmigen - hier wird die Idee zentripetaler wie zentrifugaler Kräfte im Kreis (vgl. Zinner nAB 411) wieder latent spürbar.

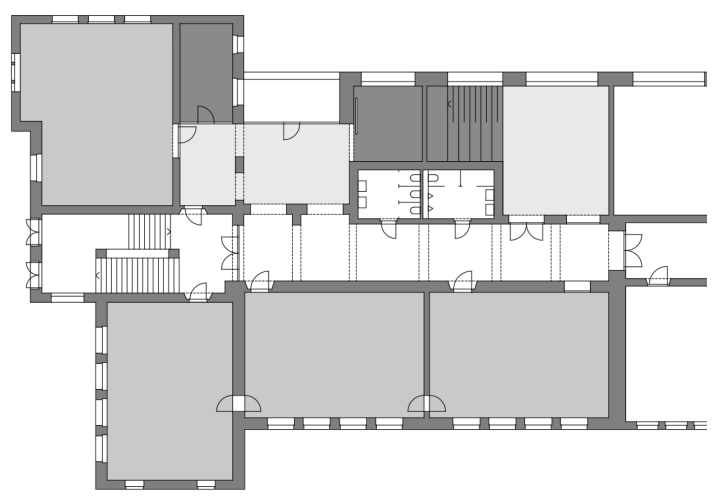

ABB 08

Gruppen (dunkel), Klassen (mittel), „Mitten“ (hell) @2020 schulRAUMkultur

Durch die zentrale Lage der WC-Gruppe und der damit verbundenen Bezugslosigkeit von Marktplatz und Bibliothek erhält das Gefüge den Charakter eines Dipols zweier Mitten (ABB 09). Links der Marktplatz und rechts die Bibliothek. Durch die Lage der Türen allerdings gewinnt der Marktplatz in Bezug auf die Klassenräume mehr "Zentralität" als die Bibliothek. Dennoch kann nicht von einem „zentrierten Cluster", sondern von einem „annähernden Cluster" gesprochen werden. Der Marktplatz selbst ist zwar ausgewiesen und benannt, doch ist er einerseits 
mit $31 \mathrm{~m}^{2}$ klein und andererseits „beschäftigen" inn reihum Blicke und Wege. Das kann das Gefühl von „Rast- und Haltlosigkeit" wecken, wird allerdings durch zwei räumlich gut angebundene Gruppenräume, die als Buchten des Marktplatzes erlebt werden können, teilweise kompensiert. Mit entsprechenden Möbeln, die auf die besondere Situation angemessen reagieren, kann aus der Wege-Not eine Verweil-Tugend werden und aus dem Namen "Marktplatz" gewissermaßen Programm. Als wohnbar gemachte Drehscheibe für Informationen, Besprechungen, Wege und Blicke ist dieser Raum dann geeignet - er wird eine „annähernde Mitte".

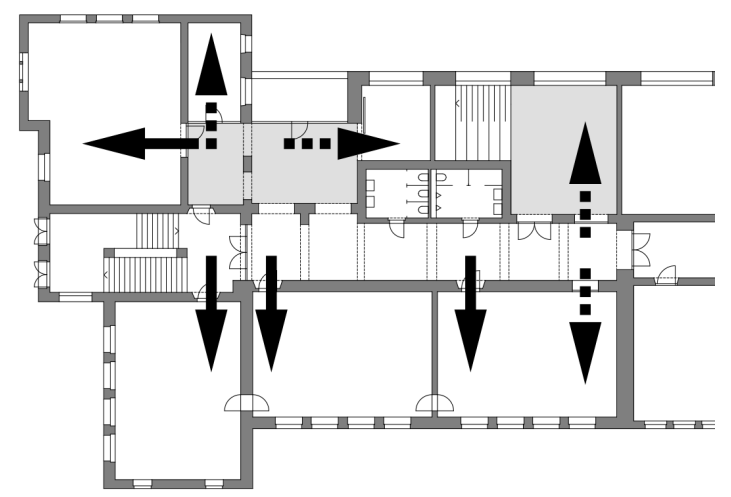

ABB 09 potenzielle Beziehungen der zwei Mitten @2020 schulRAUMkultur

Die Vervielfachung von Öffnungen zwischen Klassenräumen und hin in die Mitte (ABB 07) zeigt eine erhöhte „Beziehungsfähigkeit“ aller Räume. Zusätzlich gleicht hier kein Klassenraum dem anderen: Der - geometrisch - am periphersten gelegene (rechts) ist visuell gut zur Bibliothek angebunden; der mittlere ist mit drei Türen je nach pädagogischem Wollen dreifach orientierbar. Der quergelegte (links) - und durch den Umstand des vorgelagerten Stiegenhauses real am periphersten gelegene - Klassenraum kann am explizitesten sein Eigenleben führen und sich mit zwei Türen dennoch am gemeinsamen Geschehen anbinden. Und schließlich geht der den ersten dreien am Gang gegenüberliegende Klassenraum (oben) mit dem Marktplatz und seinen Gruppenräumen beinahe eine Symbiose ein. Solche „annähernde Cluster" fordern genaue pädagogische Re-Aktionen, die im Idealfall die Stärken des Gefüges und die Bestimmtheit der Wanddurchlässigkeiten nutzen. Die Möblierung der Mitte wird hier in jedem Fall eine entscheidende Rolle spielen. 


\section{Landeck-Angedair 2018}
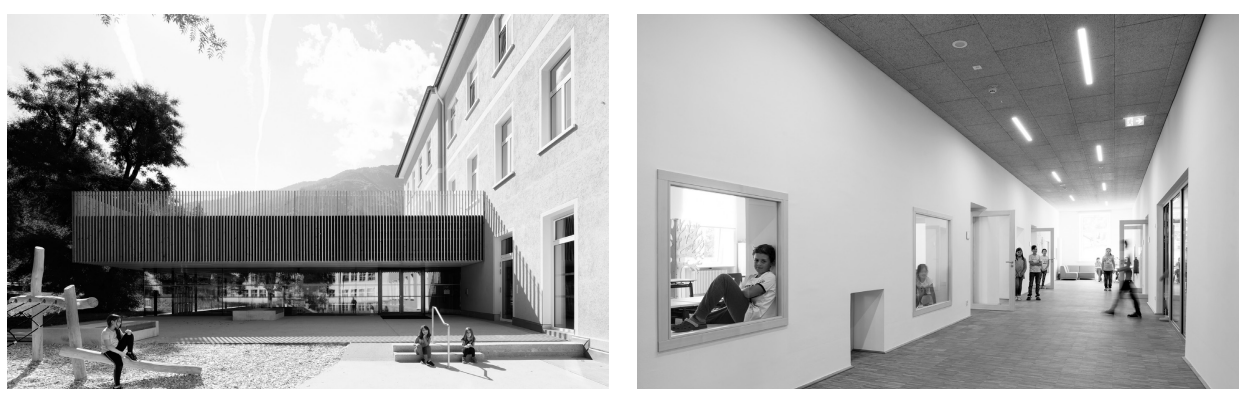

ABB 10 neuer Turnsaal, „atmungsaktiver“ Mittelgang @2018 Franz\&Sue / Lukas Schaller

Umbau und Sanierung der Volksschule im Ortsteil Angedair von Landeck in Tirol (ABB 10) wurden 2018 inklusive einer Erweiterung um einen Turnsaal fertiggestellt (vgl. Pezzei 2020; Spannberger O. J. a). Im Blick auf den gesamten Grundriss in den Obergeschoßen zeigt die bestehende Schulanlage mit eindeutigen Vorder- und Rückseiten einen effizienten Mittelgang mit zwei kopfendig gelegenen Stiegenhäusern (ABB 11 rechts). Der Abbruch des mittleren Bauwerks und das Füllen der Lücke an dieser Gebäudeseite ermöglichen Franz und Sue Architekten (www.franzundsue.at; bAIK 2014) das Versetzen beider Treppen und damit den Gewinn beider Gangenden als „annähernde Mitten“ zur Cluster-Bildung (ABB 11 mitte). Hier führt die Analyse des Bestands zu einer entschiedenen Gewichtung von Finanzmittel im Bau (Treppen).
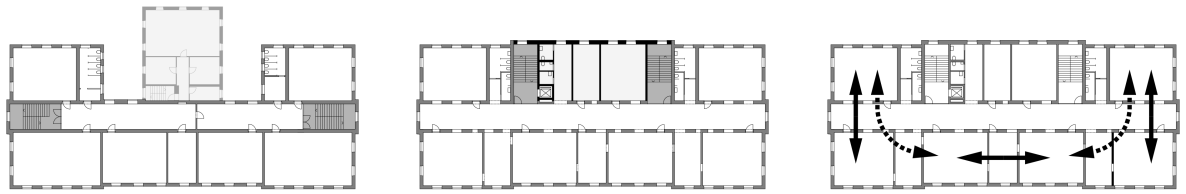

ABB 11 Abbruch, Neubau, drei Tandems oder zwei Triple @2020 schulRAUMkultur

In den neuorganisierten Geschoßen gelingt so ein schön einfach wechselnder Duktus von Lernraum und Gruppenraum. Zu diesem gesellen sich beide beruhigten Kopfenden, womit sich zu den sechs Lernräumen insgesamt fünf „Zwischenräume" als omnipräsentes und damit inklusives Raumangebot anbieten (vgl. „Klassenraum plus“ bei Montagstiftungen 2017, 27). In weiterer Folge ist jetzt sowohl die Entscheidung für drei Lernraum-Tandems (drei Vollpfeile) als auch für zwei Lernraum-Triple (zwei Halbpfeile) möglich (ABB 11 links), was für das pädagogische Geschehen einen großen organisationalen Spielraum in der Führung der Schule bedeutet. 
Im Bereich des Neubaus in der Gebäudemitte befinden sich zwei Fachräume (etwa für unterschiedliche Religionseinheiten), daneben die neuen Treppen und die Toiletten sowie gegenüber ein weiterer allgemeiner Gruppenraum. Die vielen „Räume und Räumchen“ ermöglichen ein wechselndes pädagogisches Agieren in Zeitabschnitten (täglich, wöchentlich, monatlich, halbjährlich, jährlich), etwa wenn es darum geht, personalisiert zu arbeiten, einen Teamraum in den Cluster zu verlegen oder von Triple auf Tandem umzusteigen. Das System zeigt, wie durch das Versetzen der Stiegen und das Einfügen von lediglich einer - in der Abbildung schwarz gekennzeichneten - Wand (ABB 12) ein äußerst robust kombinierbares Raum-Gefüge mit wechselseitig zuordenbaren Gruppenräumen im (in den) Cluster(n) entstehen kann.
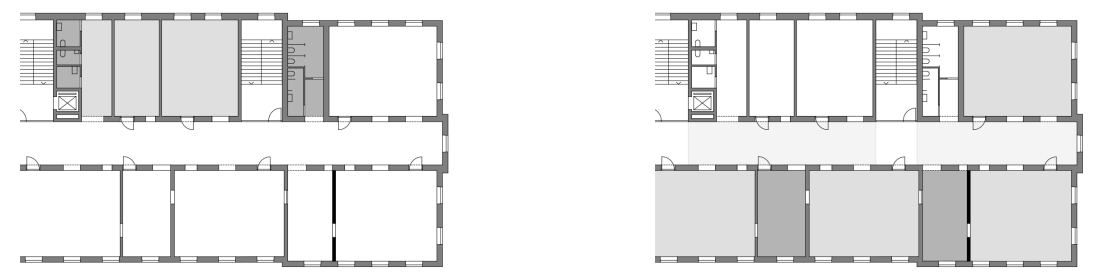

ABB 12 zentrale Räume (links), Clusterfiguren (rechts) @2020 schulRAUMkultur

Die für die Pädagogik wichtige „osmotische Qualität" der Wände wurde in diesem Projekt entschieden gesteigert: Die ehemals vier Einzel-Türen in Räume sind auf nun sieben mit zusätzlichen zwölf Fenstern ergänzt worden - hinzu kommen noch Türen und Fenster zwischen den Räumen (ABB 13). Damit wird nicht zuletzt auch dem Verlust von Tageslicht am Mittelgangstreifen etwas entgegengesetzt. Die Vielzahl der Öffnungen trägt auch zu einer Heiterkeit bei, weil sie vertikal versprungen gesetzt sind und so auf allen Höhen für Augenhöhe sorgen können.
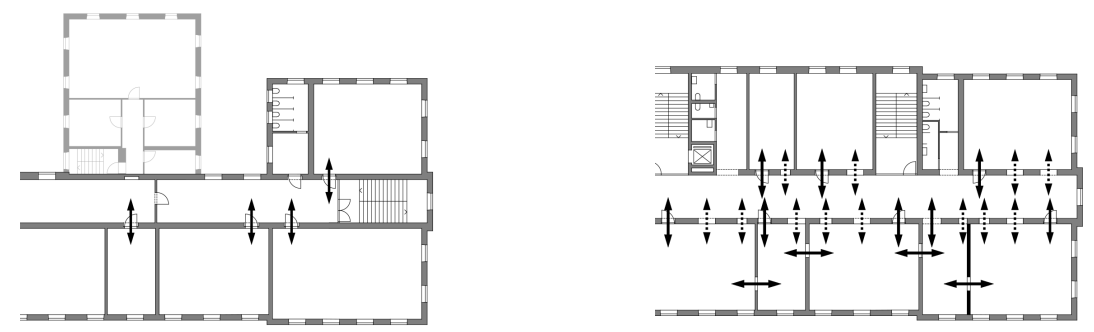

ABB 13 einzelne Türen explodieren in ein Universum an Bezügen @2020 schulRAUMkultur

In Angedair werden drei ehemalige Klassen je Geschoß-Kopfende nicht zu einem „zentrierten Cluster", sondern zu einem „annähernden Cluster" zusammengefasst. Dimension und Lage der "annähernden Mitte“ entsprechen eher einem Gruppenraum. Durch die Omnipräsenz heite- 
rer "Augen” spiegelt das Bauwerk in gewisser Hinsicht das Wesen „inklusiver Umsicht". Insofern gewinnt diese Konstellation an Tauglichkeit für den abwechslungsreichen pädagogischen Alltag einer bekanntermaßen „wachen Schule". Zusätzlich stehen ein Drittel der Fenster mit Arbeitsflächen ausgestattet und ein Set eigens entwickelter modularer Mikromöbel bereit. Das Bauwerk und seine Einrichtung wirken in Summe evokativ. In Angedair spielen Gefüge-Geometrie, Wand-Durchlässigkeit und Einrichtungs-Intelligenz (viele Eigeninitiativen!) Hand in Hand. Dieser Umstand bewirkt die spezifische Qualität eines „oszillierenden Clusters" mit annähernd zentrierendem Charakter in Teilbereichen.

\section{Leoben-Donawitz 2016}
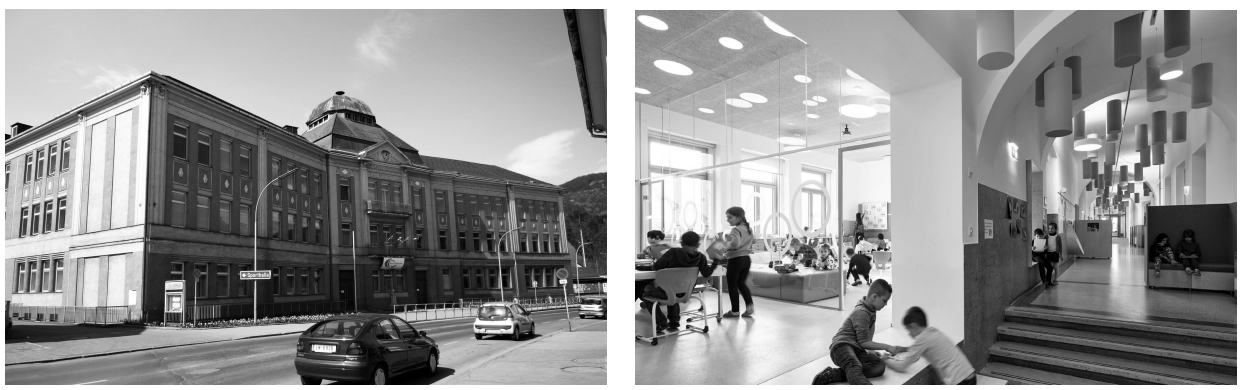

ABB 14 vor dem Umbau @2013 Stadt Leoben / eine neue Mitte @2016 Kurt Hörbst

Die Volksschule in Leoben-Donawitz wurde im Zuge der Generalsanierung des mächtigen Bildungszentrums Pestalozzi (ABB 14 links) mit Mittelschule und Polytechnischer Schule 2016 fertiggestellt. Das Forschungsteam von schulRAUMkultur \& nonconform (Zinner 2018) hat die bestehende, regelmäßige Architektur von zweimal fünf je $70 \mathrm{~m}^{2}$ groBen Klassen je Geschoß (ABB 15) zum Anlaß genommen, ein robustes System von schaltbaren Zuordnungen zu entwickeln (vgl. Kühn 2017; Zinner 2017; Spannberger o. J. b). Dieses transformiert die ehemals serielle Monotonie der Riesen-Gangschule in angemessen gestufte Hierarchien eines Bildungszentrums. In allen drei Geschoßen und in sechs seitlichen Gebäude-Flügeln, also an allen sechs „Armen” des Komplexes, gliedert der jeweils mittlere Klassenraum zwei sogenannte "Tandems“ (vgl. „Tandem“ bei Montagstiftungen 2017, 27). Diese neuen Mitten (ABB 14 rechts) in den Knickpunkten der Gänge beziehen sich auch auf die jeweilige Geschoß-Hauptmitte, diese wiederum via Luftraum über alle Ebenen auf das Zentrum im Erdgeschoß. Es entsteht ein Raumsystem von Bezügen für sechs "Schulen in der Schule" mit je rund 100 Personen (ABB 16). Dieses System ermöglicht es den drei Institu- 
tionen gewissermaßen je Zug mit vier Klassen einen Gebäude-Flügel zu bewohnen. Durch den Anbau eines vierten Stiegenhauses (rechts oben) kann dieses Gefüge alle Auflagen der Brandschutztechnik erfüllen (gering dimensionierte Abschnittsflächen, zwei Fluchtwege).

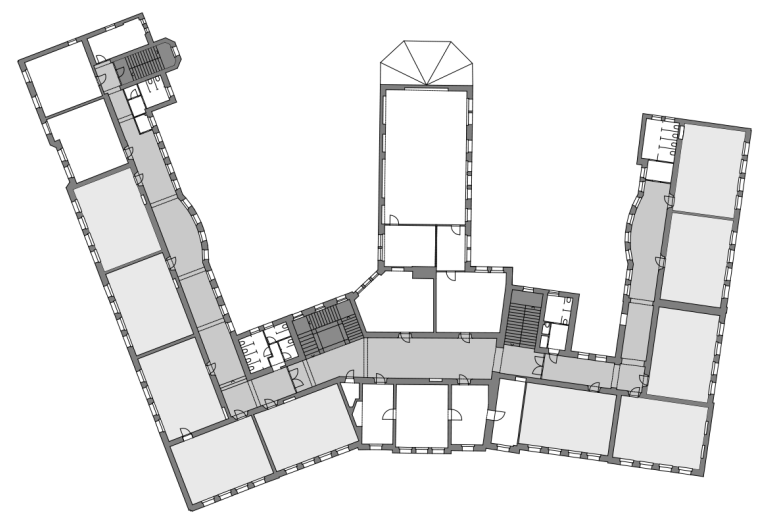

ABB 15 serielle Monotonie mit Gang und drei Stiegenhäusern @2020 schulRAUMkultur

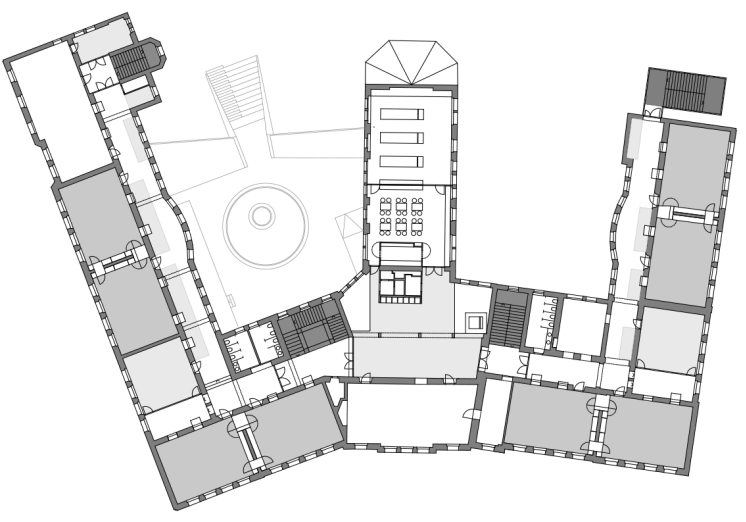

ABB 16 von serieller Monotonie zu gestuften Hierarchien @2020 schulRAUMkultur

Das bestehende System wies jeder Klasse eine Türe zu, was in den endIosen Gängen alle zehn Meter zu einem „Türereignis" führte. Im Zuge des Umbaus wurde jede Klasse mit zwei zusätzlichen Fenstern zum Gang hin geöffnet, wobei die Gestaltung Praxisrelevanz mitgedacht hat (Fensterbank in Sitzhöhe, kreisrunde Öffnungen mit Holzschaufel zum Ausruhen). Insgesamt vervierfacht sich die "Lochanzahl" in den Wänden von fünf auf zwanzig (ABB 17). Weiter werden je zwei Klassen mit zwei Türen durch eine gemeinsame tiefe Schrankwand (mit Waschbecken und Lagerflächen) zu einem Tandem zusammengefasst. Alle Möbel sind mit Rollen ausgestattet, also einfach verschiebbar. Wandöffnungen und Rollmöbel ermöglichen auf diesen nun „inklusiv umsichtigen" Flächen ein einfaches Umschalten von Intruktionsphasen 
zu klassenübergreifendem Arbeiten, zu Arbeit in Kleingruppen oder zu Einzelarbeit.
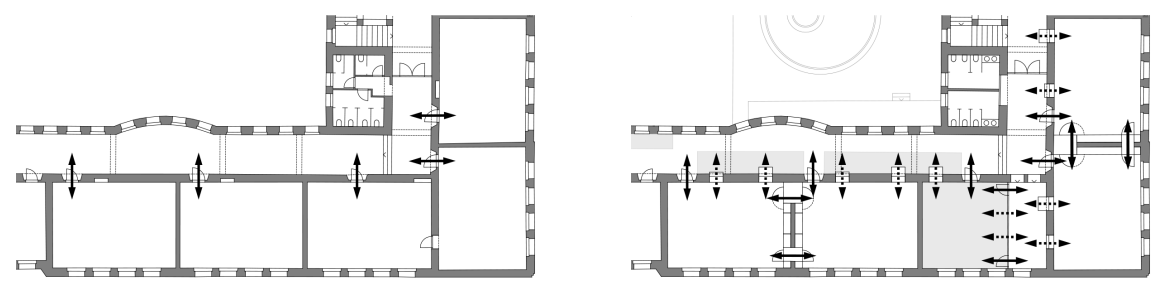

ABB 17 von einer Tür zu drei Türen und zwei Fenster je Klasse @2020 schulRAUMkultur

Die mittlere der fünf Klassen wird im Drittelpunkt mit einer raumgroßen Glaswand (samt Vorhängen) in einen Raum für Gruppenarbeit und einen Raum vor der Eck-Klasse gegliedert, der zur Gänze an das Gangsystem angebunden ist (wandgroßer Druchbruch) und dieses an seiner ehemals dunkelsten Stelle mit Licht und Sicht versorgt. Nicht nur wegen seiner Lage, sondern auch mit seinen drei Türen in Glas- und Massivwand nimmt der Gruppenraum den Charakter einer Mitte an.
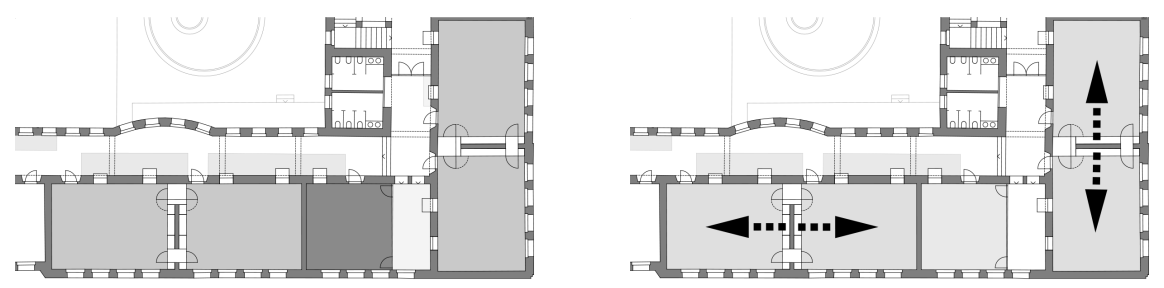

ABB 18 links Mitte (dunkel), rechts Tandems (Halbpfeile) @2020 schuIRAUMkultur

Die vier Klassenräume sind nun unterschiedlich im System eingebunden. Der Eck-Raum steckt gewissermaßen nahe an der Mitte und ist somit der exponierteste der vier. Sein Tandem-Nachbar liegt als einziger "um die Ecke" und den Toiletten gegenüber, doch hat er auch unmittelbaren Zugang zum Geschoß-Zentrum. Und schließlich liegen die beiden anderen Klassenräume gleichrangig an der langen, doch durch eine konvexe Ausbuchtung dezent entschleunigten Gangflucht. Insgesamt sind die zwei Räume der Tandems im Gegensatz zur zweigeteilten Gesamtgruppe unmittelbar erfahrbar. Das System ist trotz eines Knicks in seine Länge gedehnt, weswegen nicht von einem "seitigen Cluster", sondern von einem "annähernden Cluster" gesprochen werden kann. Auch der Umstand, dass die Mitte lediglich „annähernd“ zentripetale Kräfte entwickeln kann, spricht dafür. 


\section{die Unterschiede}

"Annähernde Cluster" zeigen im zeitgenössichen Umgang mit Gangschulen Umbau-Lösungen für eine „beweglichere“ pädagogische Praxis. Sie bieten nicht die expliziten Möglichkeiten eines „zentrierten Cluster" oder "seitigen Cluster", doch sie nähern sich diesen Lösungen auf unterschiedliche Weise und in unterschiedlichem Grade an. Ob sie alle Elemente eines Cluster-Systems (Mitte, Lernräume, Gruppenräume, Teamraum, Nebenräume) aufweisen oder nur Teile davon, hängt jeweils von strukturell-geometrischen (Gefüge) und baulich-konstruktiven (Wände) Bedingungen ab. Bei allen Kompromissen, die hier beim Clustern gefunden werden müssen, bleiben immerhin geräumige Lernräume zwischen $62 m^{2}$ und $71 m^{2}$ bestehen. In Grundschulen bildet das prinzipiell Sinn (noch starker Bezug der Kinder zum „eigenen Nest"), in Mittelschulen entspannt es immerhin die Situation unter Pubertierenden.
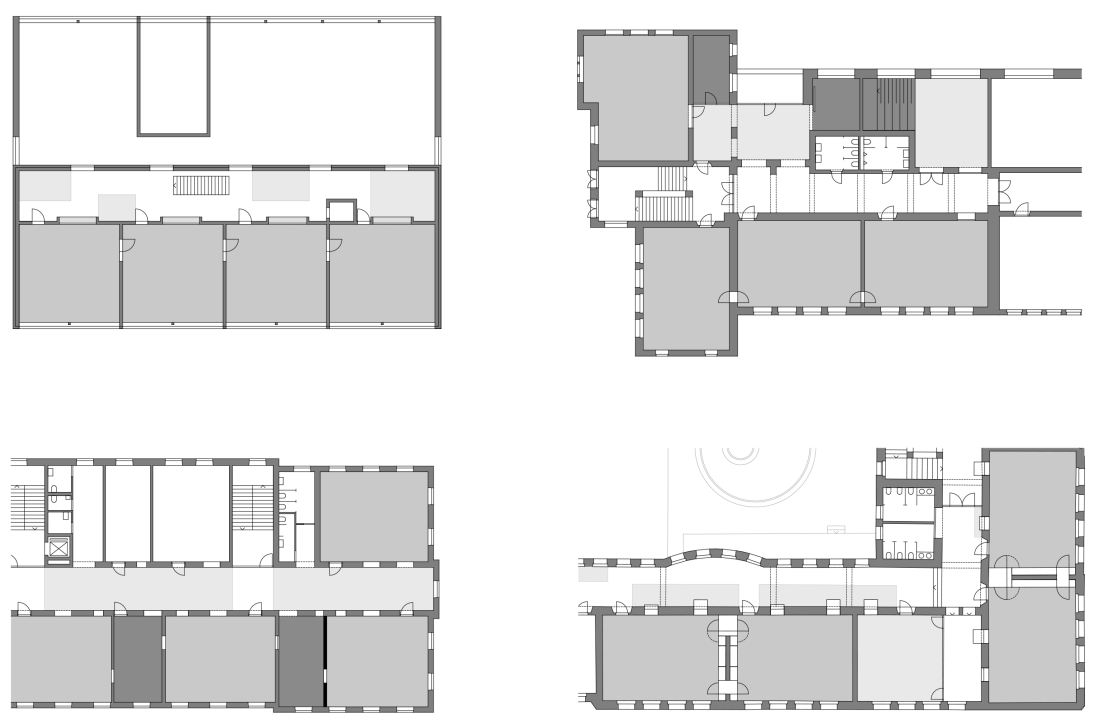

ABB 19 Mitten (hell), Klassen (mittel), Gruppen (dunkel) @2020 schulRAUMkultur

In Andelsbuch finden sich noch keine anderweitigen Räume und keine explizite Mitte, doch ein offener und integrierender Charakter der ehemaligen, nun Lernlandschaft genannten, Gangfläche. In Deutschfeistritz sind, wenn die Bibliothek als dezentraler Teamraum eingesetzt wird, zwar alle Räume eines Cluster vorhanden, doch deren Größen nicht immer adäquat genug. Hier nimmt die Gangfläche abschnittsweise zentral bindenden Charakter an (wenn akustisch noch, wie vorgenommen, nachgerüstet wird). In Angedair bietet das System alle Elemente und ist darüber hinaus im Rhythmus von Gruppen- und Lernraum gut gelokkert, doch vermag der ehemalige zu schmale Gang keinen platzartigen Charakter anzunehmen. In Donawitz kann der in der Mitte und an der 
Ecke angelegte Gruppenraum die Länge des Systems nicht entscheidend "stauchen". Neben dem Teamraum fehlt insbesondere die explizite Mitte, doch liegt eine insgesamt in Blicken und Wegen durchlässige Struktur vor.

In Andelsbuch ermöglicht die Übersichtlichkeit des Systems in Kombination mit den großzügigen Fenstern zwischen Lernlandschaft und Lernraum und mit den Türen zwischen den Lernräumen einen Flow der pädagogischen Praxis. In Deutschfeistritz können Klassen verschieden intensiv untereinander bzw. zu Mitte und Nebenräumen hin angebunden werden, es gelingt ein System unterschiedlicher Orte mit je spezifischen Chancen für den Schulalltag. In Angedair sind die hohe "Atmungsaktivität" der Wände und der rhythmisch stetige Wechsel von Lernraum und Gruppenraum Basis für eine Unzahl an Möglichkeiten der organisatorisch wie pädagogisch motivierten Nutzung. Und in Donawitz bieten die doppelt verknüpften Tandems in Kombination mit einem zwischengeschalteten Gruppenraum und breiten wie „inklusiv umsichtig" angebundenen Gängen genügend Chancen für kooperatives und klassenübergreifendes Lernen.

Der Umbau einer Gangschule zu einem „annähernden Cluster" kann also eine Verflüssigung bzw. eine Vervielfachung pädagogischer Möglichkeiten mit sich bringen. Das Ausmaß des Gelingens ist unverrückbar abhängig von der Situation im baulichen Bestand und macht einen Dialog zwischen Verwaltung und Planung (langfristige Perspektiven) sowie zwischen Planung und Nutzung (mittelfristige Perspektiven) sinnfällig. Letztlich kann die "Wendigkeit" gewählter Möbel einen entscheidenden Beitrag für den Erfolg leisten (kurzfristige Perspektiven). Jede Verwaltung, jede Schulgemeinschaft, jedes Bestandsgebäude, jedes Architekturbüro, jeder Ort und jede Zeit sind anders. Daher wird ein einfaches Übernehmen ausgetüftelter Lösungen meist nicht gelingen - auch beim Umbau von Schulen ist also der Unterschied von Rezept und Muster wesentlich, jedes Bauvorhaben in diesem Sinn ein neues. Ein spezifisches Zusammenwirken aus Geist (Schulspirit) und Körper (Schulbau) mit Knochen (Gefüge), Haut (Wände) und Innereien (Möbel) kann dann einen ganzen, insbesondere einen lebendigen Organismus ausmachen.

\section{Verbindungen}

$\rightarrow$ nAB220 von Dorfschule zu Schuldorf

$\rightarrow$ nAB410 clustern

$\rightarrow$ nAB411 zentriert clustern

$\rightarrow$ nAB412 seitig clustern 


\section{Literatur}

bAIK - Bundeskammer der Architekten und Ingenieurkonsulenten (2014): Sanierung der Volksschule Angedair, Neubau der Turnhalle Landeck. Unterlagen zu Verfahren und Beiträgen des Architekturwettbewerbs. Online verfügbar unter: http://www. architekturwettbewerb.at/competition.php?id=1413 (abgerufen am 09.11.2020).

bAIK - Bundeskammer der Architekten und Ingenieurkonsulenten (2016): Generalsanierung Schulzentrum Deutschfeistritz. Unterlagen zu Verfahren und Beiträgen des Architekturwettbewerbs. Online verfügbar unter: https://www.architekturwettbewerb. at/competition.php?id=1784 (abgerufen am 09.11.2020).

bAIK - Bundeskammer der Architekten und Ingenieurkonsulenten (2019): Sanierung und Erweiterung der Volksschule Andelsbuch. Unterlagen zu Verfahren und Beiträgen des Architekturwettbewerbs. Online verfügbar unter: https://www.architektur wettbewerb.at/competition.php?id=2415 (abgerufen am 09.11.2020).

balloon architekten ZT-OG (o.J.): Generalsanierung SZ Deutschfeistritz. Projektdokumentation auf der Website des Architekturbüros. Online verfügbar unter: http:// www.balloon-rgw.at/projekt/generalsanierung-sz-deutschfeistritz (abgerufen am 09.11.2020).

franzundsue (o. J.): Schwebend im Park. Volksschule Angedair, Landeck in Tirol. Projektdokumentation auf der Website des Architekturbüros. Online verfügbar unter: https://www.franzundsue.at/projekte/volksschule-angedair-landeck-in-tirol/ (abgerufen am 09.11.2020).

Kühn, Christian (2017): Licht von allen Seiten. Artikel im „Spectrum“ der Tageszeitung die Presse vom 11.03.017. Seite VII. Online verfügbar unter: http://www.schulraumkultur. at/perch/resources/180308-blog-kuehn.christian-170311artikel.spectrum-licht.von. allen.seiten-seitevii.pdf (abgerufen am 09.11.2020).

Montag Stiftung Jugend und Gesellschaft, Bund Deutscher Architekten BDA \& Verband Bildung und Erziehung VBE (2017): Leitlinien für leistungsfähige Schulbauten in Deutschland. Online verfügbar unter: https://issuu.com/montagstiftungen/docs/ mon_Ils_brosch_komplett_46rz_3teauf?e=17109942/50313652 (abgerufen am 08.11.2020).

Pezzei, Linda (2020): Lernen mit Leichtigkeit. In: Bildung \& Kultur. architektur Fachmagazin Ausgabe 4 / 2020. Perchtoldsdorf: Laser Verlag G.m.b.H. Online verfügbar unter: https://www.architektur-online.com/projekte/lernen-mit-leichtigkeit (abgerufen am 09.11.2020).

Robert Bosch Stiftung GmbH (o. J.): Der Deutsche Schulpreis. Portraits von ausgezeichneten Schulen. Der Schulpreis wir ausgeschrieben von der Robert Bosch Stiftung und der Heidehof Stiftung in Kooperation mit ARD und DIE ZEIT Verlagsgruppe. Online verfügbar unter: https://www.deutscher-schulpreis.de/ (abgerufen am 22.11.2020).

Spannberger, Ursula (Red.) (o. J. a): Volksschule Landeck-Angedair. Projektvorstellung im Rahmen des Schulbauatlas. Online verfügbar unter: https://atlas.pulsverbund.eu/ schule/volksschule-landeck-angedair (abgerufen am 08.11.2020).

Spannberger, Ursula (Red.) (o. J. b): Bildungscampus Pestalozzi. Projektvorstellung im Rahmen des Schulbauatlas. Online verfügbar unter: https://atlas.pulsverbund.eu/ schule/bildungszentrum-pestalozzi-leoben-donawitz (abgerufen am 08.11.2020).

stöckler gruber architekten (o. J.): Website des Architekturbüros. Online verfügbar unter: http://sg-architekten.at/ (abgerufen am 08.11.2020).

Zinner, Michael (2017): Bildungszentrum Pestalozzi. In: Franz Hammerer \& Katharina Rosenberger (Hrsg.) (2017): RaumBildung4. Wien: Hausdruckerei der AUVA, 24-45. 
Online verfügbar unter: http://www.schulraumkultur.at/perch/resources/180308blog-zinner.michael-2017artikel.raumbildung4-bildungszentrum.pestalozzi-seite 24-45.pdf (abgerufen am 09.11.2020).

Zinner, Michael (2018): Bildungszentrum Pestalozzi. Projektdarstellung im Blogeintrag vom 03.08.2018. Online verfügbar unter: http://www.schulraumkultur.at/artikel/ 2018-03-08-bildungszentrum-pestalozzi (abgerufen am 08.11.2020).

\section{Schlagworte}

annähernder Cluster, annähernd, Cluster, Nachbarschaft, Umbau, Zubau, Sanierung, schlafende Schule, wache Schule, baulicher Umbruch, pädagogischer Aufbruch, Tandem, Tripple, Mitte, Linie, Kreis, Tauglichkeit, Gangnutzung, Konfiguration, Geometrie, Haut, Beziehung, Durchlässigkeit, Möblierung, geometrische Gefügequalitäten, physiologische Hautqualitäten, unterschiedlicher Nutzungsoptionen, Gangschule, Grundschule, Volksschule, Mittelschule, Polytechnische Schule, Schulzentrum, Bildungszentrum, Volksschule Andelsbuch, stöckler gruber, Volksschule Landeck-Angedair, Franz\&Sue, Bildungszentrum Leoben-Donawitz, schulRAUMkultur \& nonconform, Schulzentrum Deutschfreistritz, balloon architekten 